\title{
Effect of Snake Venom Metalloprotease Desintegrin in Hepatocarcinoma Tumor Cells
}

\author{
Amanda Gomes'; Leticia Monica Coimbra Gaziola²; Luciana Knop³; Rosa Andrea Nogueira Laiso²; Durvanei Augusto \\ Maria ${ }^{1,2 *}$ \\ ${ }^{1}$ Medicine School of University of São Paulo; ${ }^{2}$ Laboratory of Molecular Biology, Butantan Insitutite; São Paulo, São \\ Paulo; ${ }^{3}$ Health Institute of Technologies - SENAI-CIMATEC; Salvador, Bahia; Brazil
}

\begin{abstract}
Hepatocellular carcinoma is the third leading cause of cancer-related death in the world. This cancer is associated with cirrhosis following the hepatitis $\mathrm{B}$ or $\mathrm{C}$ virus infection, alcohol addiction, metabolic liver disease and exposure to dietary toxins such as aflatoxins and aristolochic acid. Studies demonstrate the integration of the HBV genome into liver cell DNA, including cases of patients with HBV-negative serology. Despite advances in prevention techniques, screening, and technology in cancer diagnosis and treatments, the incidence and mortality remain worrisome. Therefore, this research is significant due to the contribution of the development of new biological agents that can be used as monotherapy or adjuvant chemotherapy. Jararhagin, a snake toxin isolated from Bothrops jararaca venom, has been the subject of many studies seeking alternatives for the treatment of cancer. This protein contains the cysteine-rich disintegrin-like metalloproteinase domains and desirable functions to combat tumor cells, such as promoting acute inflammation, damaging the vascular endothelium through the zinc-dependent catalytic domain (responsible for hemorrhagic function) and enzymatically degrading the constituents of the endothelial basement membrane. Due to the antitumor effects of jararhagin presented in previous research, this study aimed to describe the possible antitumor effects of this snake metalloprotease in the murine liver tumor, intending to propose a new therapeutic option in the human liver tumor.
\end{abstract}

Keywords: Cancer. HCC. Hepatocellular. Jararhagin. Metalloprotease.

Among the primary liver tumors, the most common is hepatocellular carcinoma (HCC), an aggressive disease that occurs in more than $80 \%$ of liver cancer cases [1]. HCC is the third leading cause of cancer-related death in the world. Although there are advances related to prevention techniques, screening, technology in diagnosis and treatment of cancer, the incidence and mortality remain worrisome $[2,3]$. In western countries, including Brazil, $70 \%-80 \%$ of HCC cases are associated with cirrhosis following chronic hepatitis B (HBV) or C (HCV) infection. This cancer is significantly linked to cases of $\mathrm{HBV}$ infection due to genome integration from the virus to the hepatocyte's DNA. Still, there are patients with hepatitis B virus-negative serology and HBV in their tumor. Alcohol is also a factor

Received on 24 September 2019; revised 11 October 2019. Address for correspondence: Prof. Dr.Durvanei Augusto Maria. Institute of Butantan, Biology Molecular Laboratory, Avenida Vital Brasil, Number 1500, Zip Code: 05503-900, São Paulo, SP, Brazil. Phone/Fax: (5511)2627-9750. E-mail: durvanei@usp.br

J Bioeng. Biotech. Appl. Health 2019;2(4):112-122.

C 2019 by SENAI CIMATEC. All rights reserved. associated with predisposition to liver cirrhosis and, consequently, to $\mathrm{HCC}$, as $80 \%-90 \%$ of patients with $\mathrm{HCC}$ have cirrhosis regardless of this etiology [4]. In the United States, alcohol consumption is a relevant risk factor, since the country reports alcohol abuse as a cause related to the development of the disease five times more than hepatitis $\mathrm{C}[3,5]$.

In the treatment of $\mathrm{HCC}$ in advanced stages, the indication of sorafenib, a tyrosine kinase inhibitor, is well advised. However, its use as an adjuvant after partial hepatectomy for HCC is not yet wellestablished. A randomized clinical trial comparing sorafenib to placebo involving 1,114 patients on adjunctive therapy after partial hepatectomy or HCC ablation was recently completed. In this study, 900 patients received surgical treatment for HCC. However, this trial demonstrated that there is no statistical difference in survival between the group of patients receiving sorafenib and the control group [5].

Liver cancer survival rate is up to 5 years after diagnosis [6], following the same relative 5-year survival for all cancers. Data reveal that cancer survival has increased significantly over the past 
three decades, in part due to targeted therapy. Nevertheless, there are still many site-specific medications that may play a role only in a portion of cancer patients who have specific molecular changes. It is necessary to continue developing new biological agents that can be used alone and/ or as adjuvant therapy in protocols with current chemotherapic drugs already approved by the competent organs [7].

In this context, jararhagin (snake toxin isolated from Bothrops jararaca venom) has been the subject of many studies seeking alternatives for the treatment of cancer. This protein contains the cysteine-rich disintegrin-like metalloproteinase domains and desirable functions to combat tumor cells, such as promoting acute inflammation, damaging the vascular endothelium through the zinc-dependent catalytic domain (responsible for hemorrhagic function) and enzymatically degrading the constituents of the endothelial basement membrane $[8,9]$.

A study of jararhagin-treated cell culture macrophages showed increased expression of proinflammatory mediators, including IL-1, a cytokine that induces inflammation associated with expression of adhesion molecules such as CD44 invasion and migration marker, CD34 angiogenesis marker, increased TNF-alpha, crucial in the development of jararhagin-induced necrosis, and the release of chemotaxis mediators [10].

In other study, low-dose jararhagin-induced effects were antiproliferation and induction of cell death of SK-Mel-28 lineage melanoma cells, which was also supported by increased expression of cell cycle-related genes and apoptosis [9]. Jararhagin also showed cytotoxicity in breast tumor cells. Promoting alterations in the cell cycle, death of tumor cells by necrosis, even due to high inflammatory intensity, reduced viability of tumor cells [10].

Due to the antitumor effects of jararhagin presented in previous research, this study aimed to elucidate the possible antitumor effects of this snake venom metalloprotease in the murine liver tumor, aiming to propose a new therapeutic option in the human liver tumor.

\section{Methods}

Monolayer Cell Culture

HEPA-1c1c7 murine hepatocarcinoma cell lines, code CRL-2026 originating from the American Type Culture Collection (ATCC), were used, maintained, and stored in the cell bank by Dr. Durvanei Augusto Maria (Biology Molecular Lab., Butantan Institute). After thawing, the cells were transferred to a cell culture bottle (25 $\mathrm{cm}^{2}$ ) containing DMEM and MEM- $\alpha$ culture medium (Cultilab, Campinas-SP), respectively, supplemented with $10 \%$ fetal bovine serum, 200 $\mathrm{mM}$ sodium bicarbonate, $\mathrm{pH} 7.4$ in an oven 5\% $\mathrm{CO}_{2}$ at $37^{\circ} \mathrm{C}$. Monolayer cells were subjected to enzymatic dissociation with $0.2 \%$ trypsin solution $+0.02 \%$ EDTA to detach the cells. Enzyme neutralization was performed using the same DMEM culture medium containing 10\% SFB. After neutralization, the suspended cells were counted in a Neubauer chamber, and the concentration adjusted to $10^{5}$ cells $/ \mathrm{mL}$. The Trypan blue exclusion test determined cell viability.

Determination of Cytotoxic Activity by MTT Method

Tumor cells were incubated in 96-well plates at a concentration of $2 \times 10^{5} \mathrm{cells} / \mathrm{mL}$ for 24 hours and treated with jararhagin at multiple concentrations. The culture medium was used as a Control Group to dilute the jararhagin stock solution. After 24 hours of treatment, the supernatant was collected on another plate, and $100 \mu \mathrm{L}$ of MTT (Calbiochem - Darmstadt, Germany) added at a concentration of $5 \mathrm{mg} / \mathrm{mL}$; then the cells were incubated for 3 hours in an oven containing $5 \% \mathrm{CO}_{2}$ at $37^{\circ} \mathrm{C} . \mathrm{W}$. Afterwards, the contents werere moved and $100 \mu \mathrm{L}$ of methylalcohol was added to dissolve the formed and precipitated formazan crystals. Absorbance quantification was performed in a 540 $\mathrm{nm}$ wavelength ELISA reader. Toxicity-inducing concentration in $50 \%$ of cells (IC50\%) was determined at treatment after 24 hours at different concentrations to assess the dose-response effect. The percentage of cell death, calculated concerning the negative-control, expressed the cytotoxicity of each treatment. 
Determination of Polyunsaturated Lipid Peroxidation Products

The cell culture supernatants used for the MTT cytotoxicity test and kept under refrigeration at $-20^{\circ} \mathrm{C}$. The samples were thawed at room temperature. Then $50 \mu \mathrm{L}$ of the sample was added in microtube, along with $250 \mu \mathrm{L}$ of trichloroacetic acid - 20\% TCA (Sigma-Aldrich) in other microtube, $50 \mu \mathrm{L}$ of the same sample was added with $250 \mu \mathrm{L}$ of thiobarbituric acid - TBA $0.86 \%$ (Sigma-Aldrich, Cat.: T550-0). The microtubes were placed in a water bath $\left(100^{\circ} \mathrm{C}\right)$ for 20 minutes, followed by cooling at $0^{\circ} \mathrm{C}$ for 20 minutes, centrifugation at $8,000 \mathrm{rpm}$ for 4 minutes, and the supernatant was used to quantify TBARS. The reading was performed on the spectrophotometer at a wavelength of $535 \mathrm{~nm}$.

Morphological and Marker Analysis with Confocal Laser Microscopy

An aliquot of HEPA-1c1c7 tumor cell culture grown in a 24-well plate and coverslip with $10 \%$ SFBMEM- $\alpha$ culture medium kept in the oven at $5 \% \mathrm{CO}_{2}$ at $37^{\circ} \mathrm{C}$. Cell samples from the Jararhagin-treated Groups and Control Group shave gone through a culture medium removal process, then $1 \mathrm{~mL}$ of paraformaldehyde was added to fix the cells. After this process, $1 \mathrm{~mL}$ of 2 times of PBS was added for washing, and also $300 \mu \mathrm{L}$ of bovine serum albumin (BSA) for 10 minutes, then a new PBS wash was performed. After that, $10 \mathrm{uL}$ of Triton-X was added and incubated for 1 hour. Afterward, it was added in each well-plate over the coverslips containing the cells, $1 \mathrm{uL}$ of CD44 and CD34 antibodies labeled with green fluorescein isothiocyanate (FITC) and Caspase 3, PE53 and COX-2 labeled with PE (Phycoerythrin) red fluorochrome. The plate remained overnight in the refrigerator at $-4^{\circ} \mathrm{C}$. After this process, 3 washes with 1 PBS-time were performed. Then, PBS washes were performed 3 times. The coverslips were mounted, and $3 \mu \mathrm{L}$ of the mounting medium for fluorescence protection (Fluoroshield, SigmaAldrich) were placed on them. The assembled blades were wrapped in aluminum foil to protect fluorescence and stored at $4{ }^{\circ} \mathrm{C}$ until visualization.
Scanning Electron Microscope (SEM)

Jararhagin-treated HEPA-1c1c7 $\left(5 \times 10^{5}\right)$ tumor cells were washed for 24 hours with PBS and fixed in $2.5 \%$ glutaraldehyde, $\mathrm{pH} 7.2$ at $4{ }^{\circ} \mathrm{C}$. After 24 hours of fixation, the cells were washed in a $\mathrm{Na}_{3} \mathrm{PO}_{4}$ buffer and were post-fixed in $1 \%$ osmium tethoxide for 2 hours at $4^{\circ} \mathrm{C}$. Samples were dehydrated in increasing ethanol series, immersed in isoamylacetate and critical-point dried in liquid $\mathrm{CO}_{2}$ for 5 minutes. The samples were atomized and coated with gold metal and examined on the Leo 435VP Zeiss Scanning Electron Microscope (Carl Zeiss,Germany).

\section{Cell Cycle Phases Analysis by Flow Cytometry}

After confluence in the 6-well plate, the cells were subjected to jararhagin treatment for 24 hours. The Control Group was from the same cell lineage, but not treated. The cells were then trypsinized and resuspended in PBS, centrifuged twice at $3,000 \mathrm{rpm}$ with PBS solution and resuspended in $200 \mu \mathrm{L}$ propidium iodide solution (18ug) (SigmaAldrich) containing $20 \mathrm{uL}$ Triton X-100 (SigmaAldrich) and $4 \mathrm{mg}$ RNAse-A (Molecular Probes) for 30 minutes at room temperature, protected from light. After this period, the samples were transferred to cytometry tubes, and the images were captured in a flow cytometer (FacscaliburBecton and DicKson).

\section{Results}

Determination of Cytotoxic Activity by MTT Method

After 24 hours of adhesion, in a 96-well plate, the cells were incubated, diluted in culture medium at $10 \%$ of fetal bovine serum and $1 \%$ of penicillin antibiotic with different jararhagin concentrations of $0.001 \mathrm{nM}-1 \mu \mathrm{M}$.

After treatment with jararhagin, the HEPA1c1c7 tumor cells showed morphological alterations, such as cell lysis and the formation of cell debris, from the concentration of $11.9 \mathrm{nM}$ (Figures 1, 2, 4, 5, 6). The other concentrations showed cytotoxic effects, with the percentage increase in cell mortality, loss of cell adhesion, 
cytoplasmic membrane fragmentation, and loss of cytoplasmic prolongation progression.

Determination of Lipoperoxidation (LPO) Products by Quantification of Thiobarbituric Acid Reactive Substances (TBARS) (Figure 3)

Thiobarbituric acid reactive substances (TBARs) method quantified lipid peroxidation in cell membranes and free radical formation. Malondialdehyde (MDA) is one of the end productsoflipoperoxidation formed by the primary or secondary decomposition of intermediate products, and reacts with thiobarbituric acid were widely used in the determination of oxidative stress.

Jararhagin treatment resulted in a significant formation of lipid peroxidized radicals from the hepatocarcinoma tumor lineage, with significant oxidative damage from $19.8 \mathrm{nM}$ concentrations. There is a positive correlation between the effects of jararhagin and the production of lipoperoxidation radicals. Quantification of TBARs revealed lower free radical production at high concentrations, which increased progressively with longer incubation time with jararhagin.

Morphological and Marker Analysis with Confocal Laser Microscopy

In this project, confocal laser microscopy was used to visualize morphological alterations of HEPA-1c1c7 tumor cells after 24 hours of jararhagin treatment at concentrations of $128.7 \mathrm{nM}$ and $186.2 \mathrm{nM}$.

\section{Morphological Alterations in Jararhagin-Treated} HEPA-1C1C7 Tumor Cells

Jararhagin was used to elucidate the role of the disintegrin domain of this toxin on the induced effects on liver carcinoma tumor cells as an experimental model to evaluate the possible mechanisms of inhibition of proliferation and metastases. The morphological effects on HEPA$1 \mathrm{c} 1 \mathrm{c} 7$ tumor cells were dose and time- dependent. These effects include loss of cytoplasmic expansions, round-shaped cellular aggregates, and cell detachment to the bottom of bottles and formation of apoptotic bodies, as demonstrated by scanning electron microscopy (Figure 6), similar to the cytological results obtained by Corrêa and colleagues (2002) and Klein and colleagues (2011) in human melanoma cells SKMel-28 [9,14].

The morphology was dramatically altered by treatment with the toxin. Scanning electron microscopy (SEM) showed that monolayer-grown hepatocarcinoma cells showed changes in an extracellular matrix organization with significant deposition and dense extracellular matrix synthesis when compared to untreated Control Group cells (A, B, C - Control Group - Figure 5) (D-I Jararhagin-treated Group - Figure 5). Tumor cells showed retraction and aggregates with jararhagin treatment at $128.7 \mathrm{nM}$ concentration (Figures $5 \mathrm{D}$, E, F). The $186.2 \mathrm{nM}$ treated tumor cells showed detachment of the plaque surface and formation of smaller aggregates, apoptotic bodies, and necrotic debris (Figure $5 \mathrm{G}, \mathrm{H}, \mathrm{I}$ ). Arrows show the formation of apoptotic bodies (12,000x).

\section{Cell Cycle Phases Analysis by Flow Cytometry}

The effect of jararhagin on cell cycle phases was determined after evaluating cell viability by MTT methods. The concentrations of $128.7 \mathrm{nM}$ and $186.2 \mathrm{nM}$ were selected. Cell aggregates were collected from both supernatant and adherent cells after trypsinization and evaluated by flow cytometry. Histograms show the percentage of cells distributed in the different phases of the G0 /G1, S, and G2/M cell cycle and sub-G1cells (Figure 7).

We observed that jararhagin changes the proportion of cells at different stages of the cell cycle. Figure 3 presented the value of the mean percentage \pm sd of cells in the cell cycle phases of HEPA-1c1c7 tumor cells. The jararhagin treatment induced increased populations with fragmented DNA and G2/M arrest. The 186.6nM concentration of jararagine induced a fourtimes increase in the proportion of cells in the sub-G1 phase, demonstrating their potential for cytotoxicity. 
Figure 1. Appearance of the cellular alterations presented after the jararhagin treatment of HEPA-1c1c7 tumor cells.
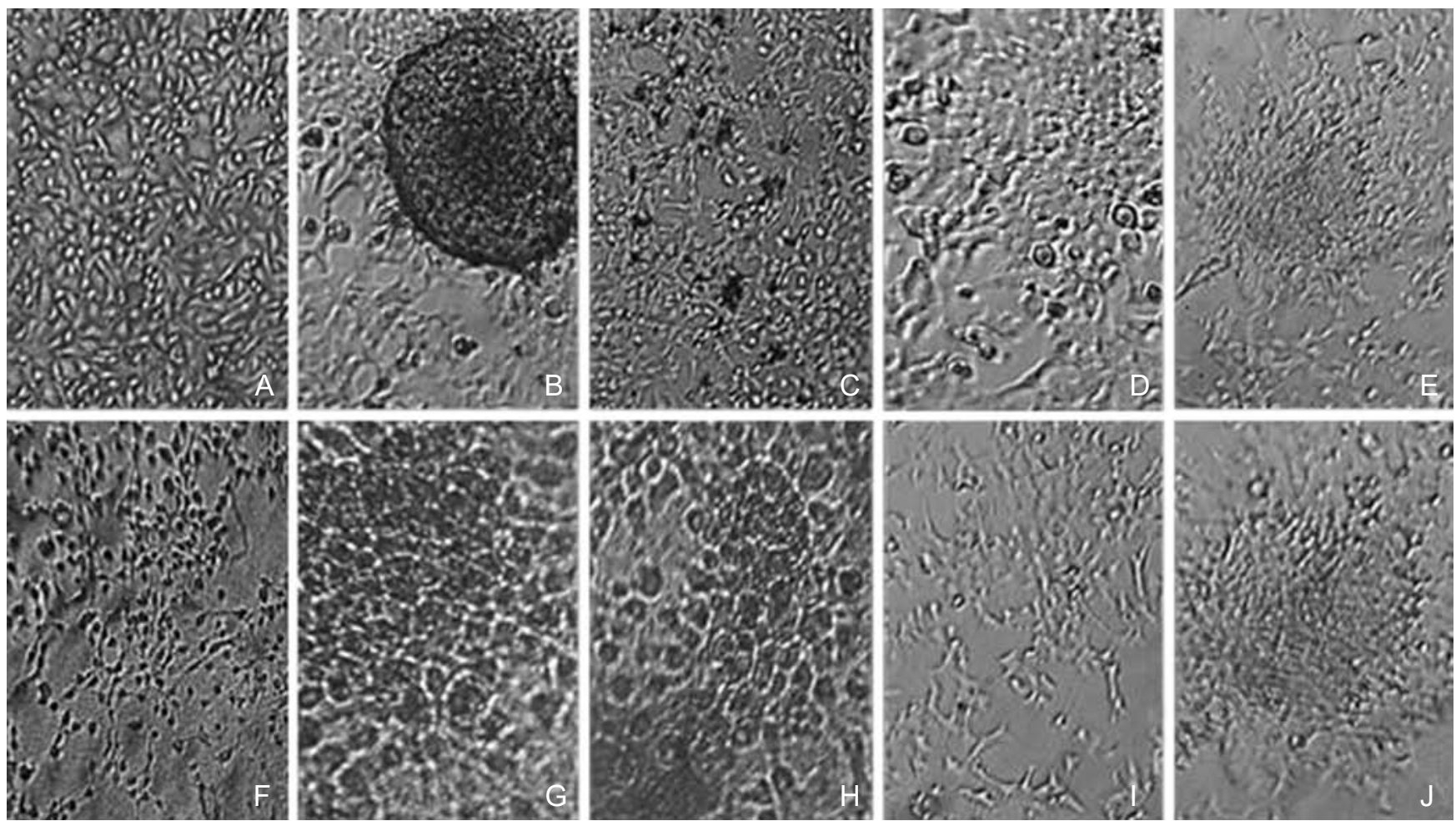

(A) Control Group. (B) Jararhagin-treated Goup with $0.001 \mathrm{nM}$ concentration. Significant changes were observed in morphology and cell adhesion. (C) Jararhagin-treated Goup with $0.001 \mathrm{nM}$ concentration presented a moderate loss of cell adhesion and morphological alterations with consequent cell death; (D) Jararhagin-treated Goup with $0.13 \mathrm{nM}$ concentration showed moderate loss of cell adhesion and morphological alterations. In the other concentrations, from $1.5 \mathrm{nM}$ to $1 \mu \mathrm{M}$, there were slight changes in cell morphology.

Figure 2. Inhibitory concentration (50\%) obtained by the equation of a line at $186.2 \mathrm{nM}$.

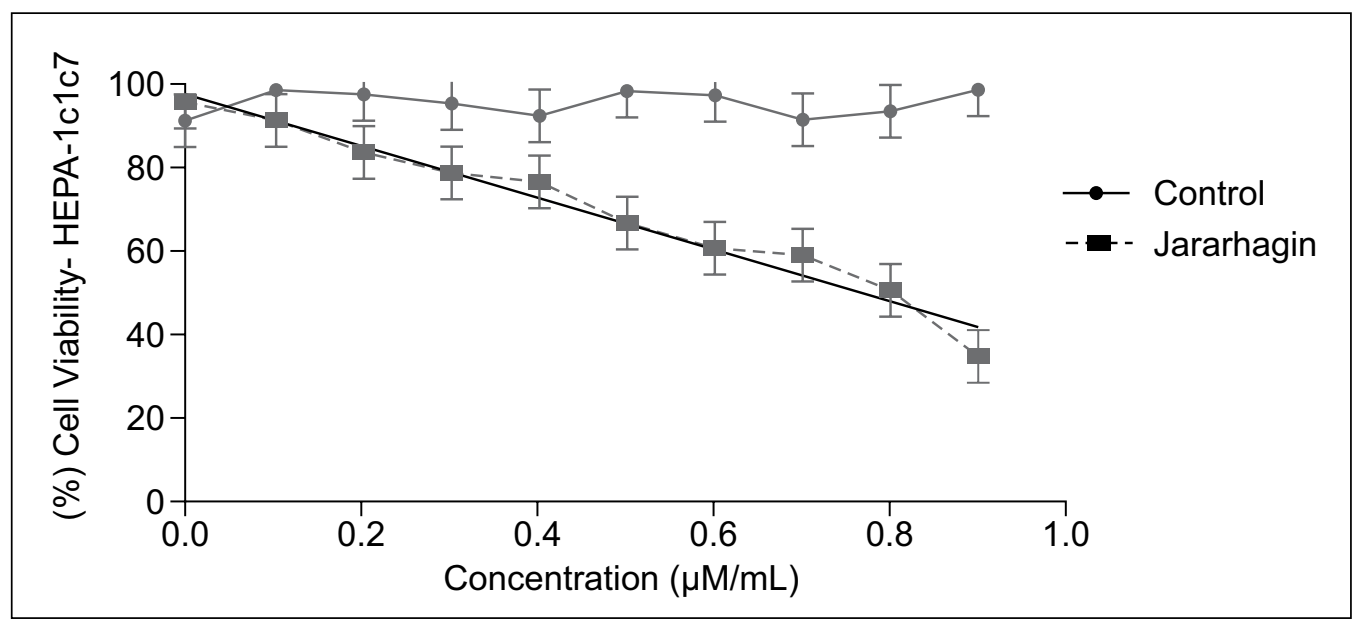

The number of experiments performed $n=8$. * Significant differences between the Jararhagintreated Group and Control Group. ANOVA Variance Test followed by Tukey Kramer's Multiple Test. $\mathrm{p}<0.001$. 
Figure 3. Mean values \pm standard deviation of malondialdehyde (MDA) lipopedoxidation.

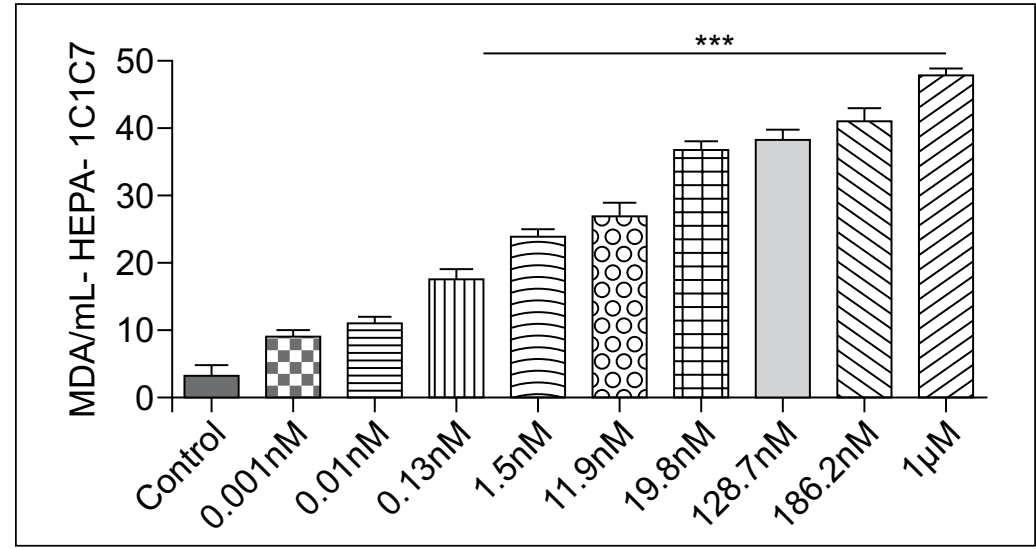

Number of experiments performed $n=8 . *$ Significant differences between the Jararhagin-treated Group and Control Group. ANOVA Variance Test followed by Tukey Kramer's Multiple Test. $\mathrm{p}<0.001$.

Figure 4. Photomicrograph of monolayer culture of HEPA-1c1c7 tumor cells acquired by confocal laser microscopy.

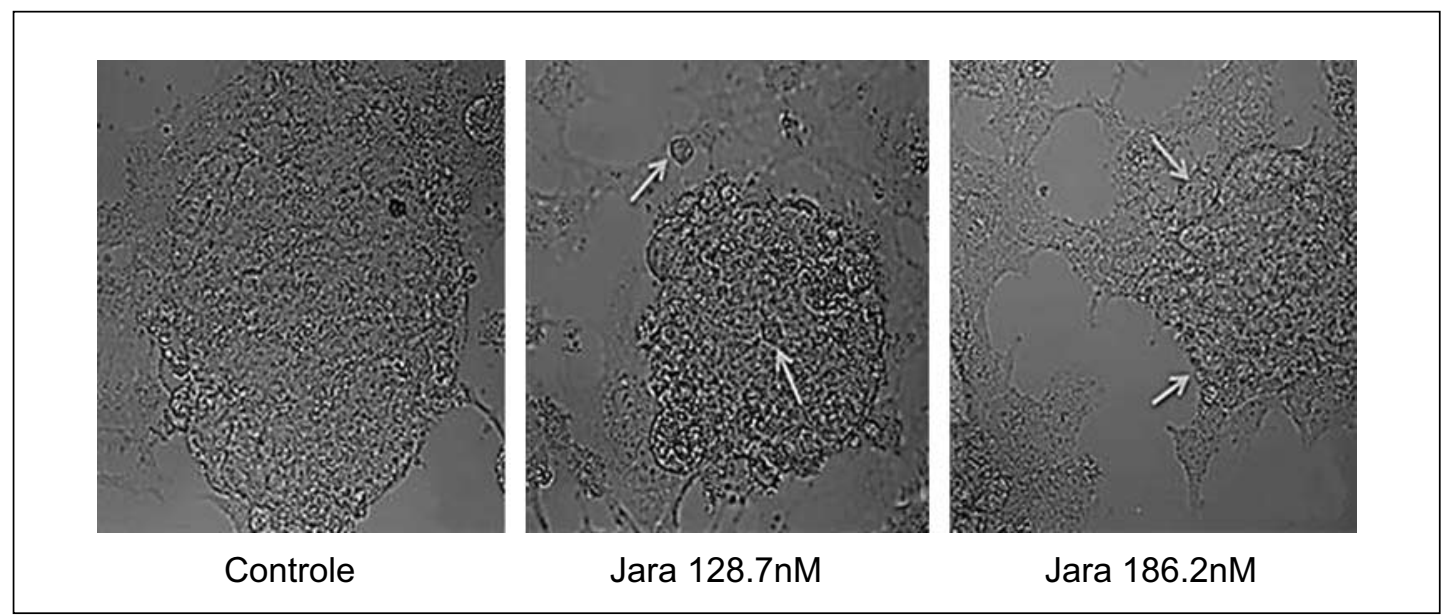

(A) Control Group - cells show epithelial morphology. (B) Jararhagin-treated Group at $128.7 \mathrm{nM}$ concentration - cells undergoing necrosis and apoptosis. (C) Jararhagin-treated Group at $186.2 \mathrm{nM}$ concentration - cells undergoing necrosis and apoptosis, with retraction of the cytoplasm and formation of apoptotic bodies. White arrows: apoptotic bodies; necrotic structures with the pyknotic nucleus.

\section{Discussion}

Several types of research involving cell cultures evaluate toxins isolated from snake venom and the effects on several tumor cell lines, aiming to find substances with possible therapeutic potential, acting as a single or adjuvant chemotherapeutic agent[11].

According to Moura and colleagues [12], jararhagin hemorrhagic activity is related to jararhagin specificity by $\alpha 2 \beta 1$ integrins expressed in endothelial cells [12]. One study demonstrated the pronounced expression of these integrins during embryonic development, as well as the fragility of capillaries that are constituted of a single layer of endothelial cells. The basal lamina appearing on the eighth day [13], a fact demonstrated by hemorrhage provoked by jararhagin in a time/dose-dependent form on this study. 
Figure 5. Photomicrograph of monolayer culture of HEPA-1c1c7 tumor cells acquired by confocal laser microscopy.

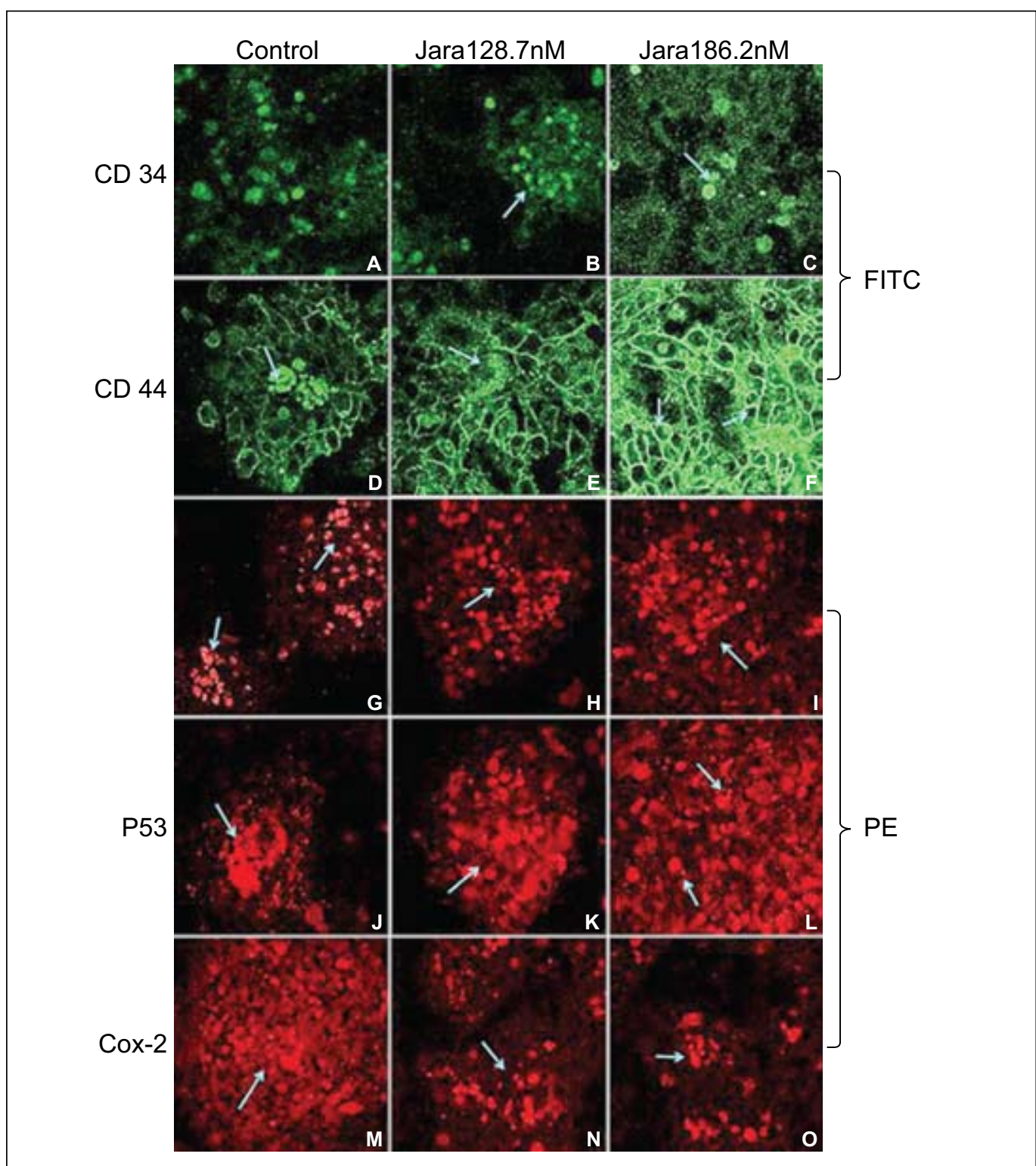

(A) CD-34 protein expression in the Control Group. (B) CD-34 protein expression in the Jararhagintreated Group at $128.7 \mathrm{nM}$ concentration - cells were undergoing necrosis and apoptosis. (C) Expression of CD-34 protein in the Jararhagin-treated Group at $186.2 \mathrm{nM}$ concentration. (D) CD-44 protein expression in the Control Group. (E) CD-44 protein expression in the Jararhagin-treated Group at $128.7 \mathrm{nM}$ concentration - cells were undergoing necrosis and apoptosis. (F) CD-44 protein expression in the Jararhagin-treated Group at $186.2 \mathrm{nM}$ concentration. (G) Caspase-3 protein expression in the Control Group. (H) Caspase-3 protein expression in the Jararhagin-treated Group at $128.7 \mathrm{nM}$ concentration cells were undergoing necrosis and apoptosis. (I) Caspase-3 protein expression in the Jararhagin-treated Group at $186.2 \mathrm{nM}$ concentration. (J) P53 protein expression in the Control Group. (K) P53 protein expression in the Jararhagin-treated Group at $128.7 \mathrm{nM}$ concentration - cells were undergoing necrosis and apoptosis. (L) P53 protein expression in the Jararhagin-treated Group at $186.2 \mathrm{nM}$ concentration. (M) Cox-2 protein expression in the Control Group (N) Cox-2 protein expression in the Jararhagintreated Group at $128.7 \mathrm{nM}$ concentration - cells were undergoing necrosis and apoptosis. (O) Cox-2 protein expression in the Jararhagin-treated Group at $186.2 \mathrm{nM}$ concentration. The arrows show points where there was a pronounced expression of each protein analyzed, respectively. 
Figure 6. Morphological characteristics of HEPA-1c1c7 tumor cells exposed to jararhagin for 24 hours at different concentrations.

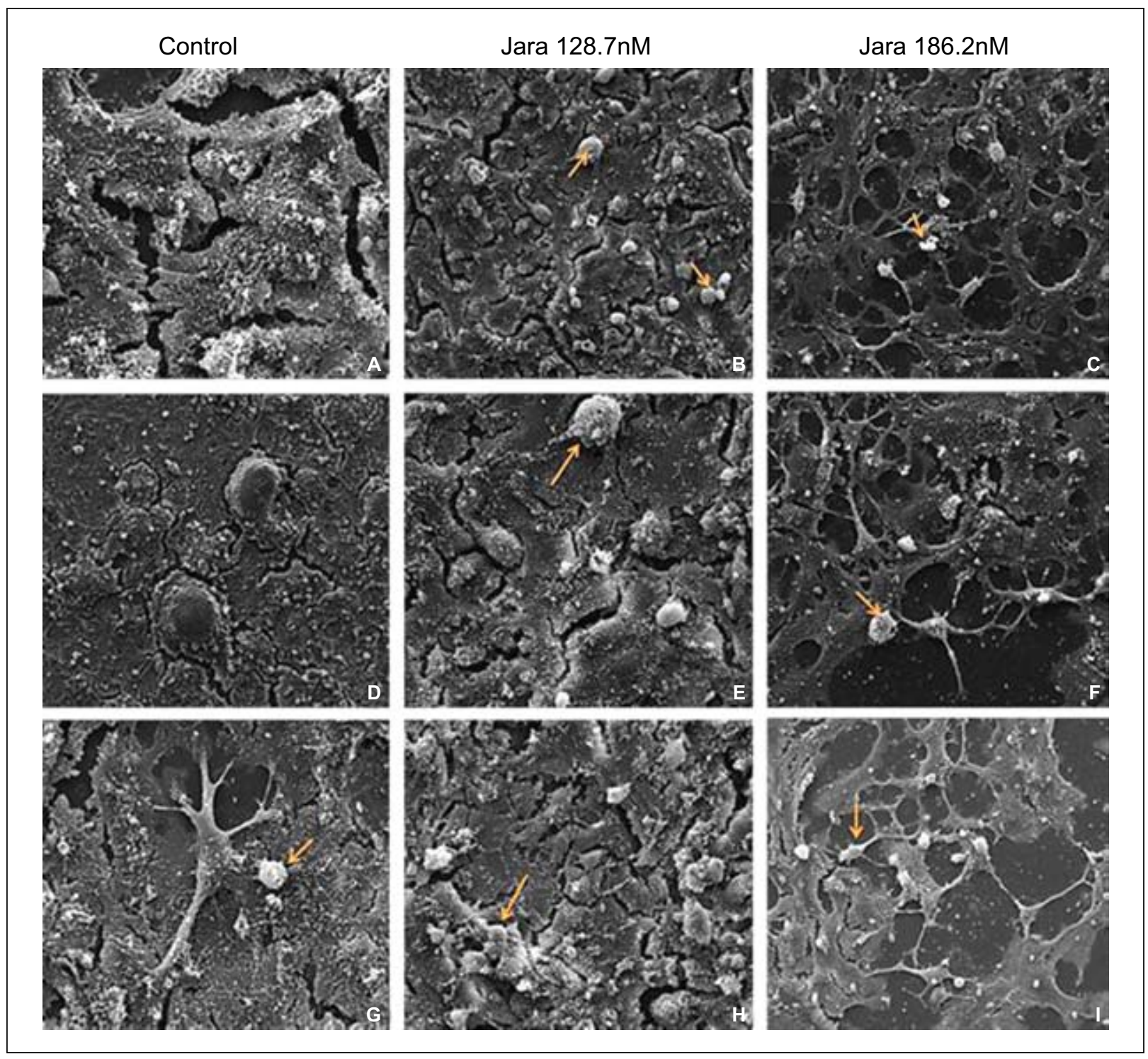

Jararhagin, isolated from Bothrops jararaca venom, induces changes in the morphology and viability of human melanoma cells (SK-MEL28 ), in addition to reducing in vivo the number of metastases when pretreated and injected in mice, according to data obtained by our research group in 2011 [9]. Besides, the jararhagin treatment also promotes morphological alterations such as cytoplasmic retraction, loss of adhesion and formation of large multicellular aggregates. These changes were accompanied by decreased expression of the CDK2 and CDK4 genes, promoting an increase in the population of cells in S-phase of the cell cycle[14].

In this study, the cytotoxic effects in HEPA1c1c7 murine hepatocellular carcinoma cells were elucidated. The cell viability evaluated after 24 showed reduced time/concentration-dependent cell viability. At $19.8 \mathrm{nM}$ jararhagin concentration, there was a significant decrease in cell viability and pronounced morphological alterations such as loss of adherence in HEPA-1c1c7 hepatocellular carcinoma cells at the $186.2 \mathrm{nM}$ jararhagin concentration. 
Figure 7. Means \pm standard deviation of the cell cycle phases of the HEPA-1c1c7 tumor cell of the Control Group and Jararhagin-treated Groups at concentrations of 128.7nM and 186.2nM.

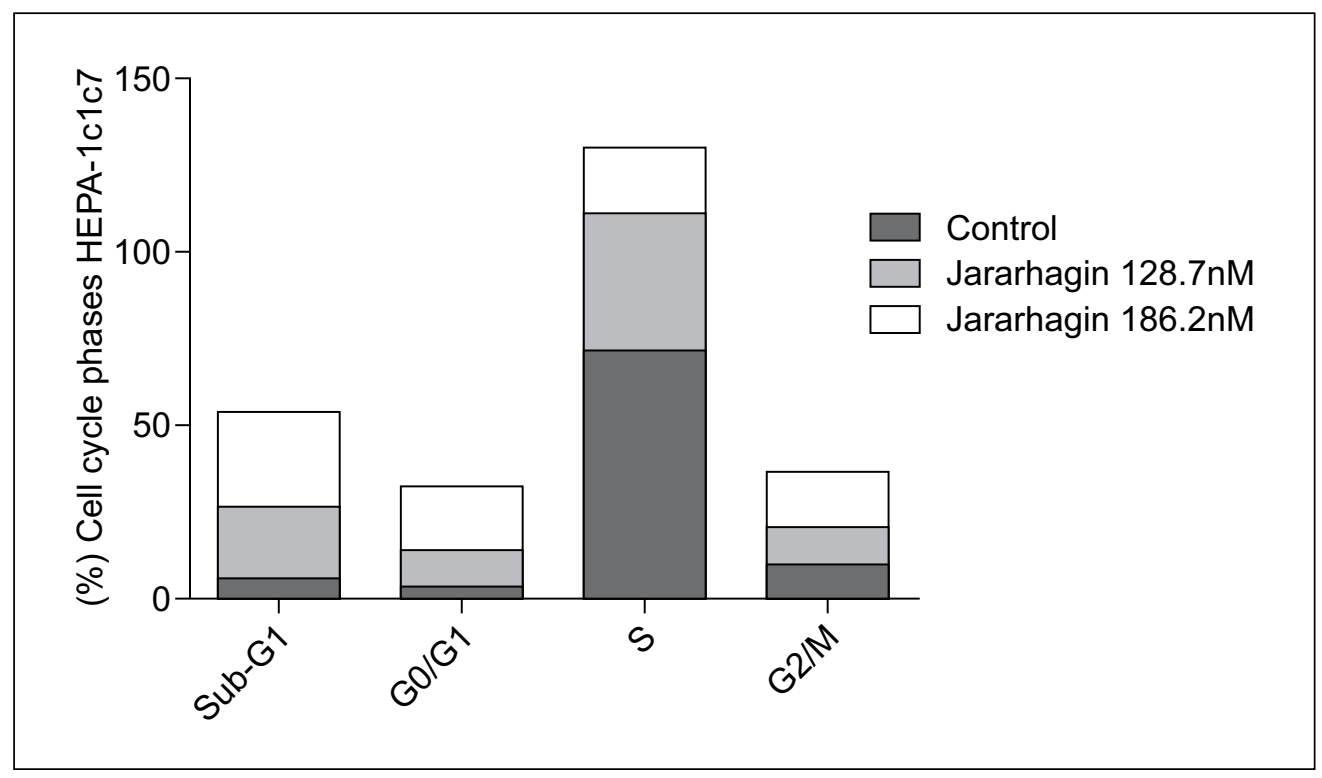

The number of experiments performed $n=06$. *Significant differences between the jararhagintreated Groups and Control Group. ANOVA Variance Test followed by Tukey Kramer's Multiple Test. $\mathrm{p}<0.001$.

MTT is a quantitative method, an indicator of mitochondrial metabolic activity, with a linear relationship between cellular activity and absorbance. Loss of membrane integrity often occurs after completing apoptosis in most of the cellular systems. Thus, treatment with jararhagin induced a decrease in mitochondrial activity at a concentration of $186.2 \mathrm{nM}$ in HEPA-1c1c7 tumor cells, and an increase in the percentage of inactive mitochondria, showing a cytotoxic effect of jararhagin.

In the process of origin of the lumen in cell culture, the selection of cells resistant to the anoikis process occurs, with the formation of cell groups and spheroids [15-16]. Spheroid growth is the result of aggregation, proliferation, polarization, adhesion molecule modulation, and lumen cell death $[17,18]$.

Reactive oxygen species (ROS) are produced continuously in cells as by products of cellular metabolism, performing essential functions as mediators of protective mechanisms such as apoptosis, phagocytosis, and detoxification [18].
Excessive increase in ROS formation induces DNA damage, promoting mutagenic alterations of nucleotides, protein oxidation, and lipid peroxidation, playing a crucialroleintumorigenesis. Free radical-induced lipid peroxidation is a widely investigated process, and the cell membrane is one of the components most affected by the high concentration of polyunsaturated fatty acids susceptible to oxidation[19].

Oxidants, in general, produce protein modifications leading to loss of function and increasing the degradation rate of oxidized proteins, promoting changes in their structure and permeability, which leads to inhibition of cell growth and death. Lipid peroxidation may trigger the apoptosis process, activating the intrinsic apoptosis pathway present in all cells, whether or not dependent on the phosphorylation of the caspase pathway. Given the importance of the lipid peroxidation process in apoptosis, the results indicate that jararhagin dose-dependently affects this process, accompanied by cellular cytotoxicity and cellular morphological alterations, inducing 
apoptosis, especially at concentrations below $1 \mu \mathrm{M}$ jararhagin in HEPA-1c1c7 tumor cells. There was also a decrease in the synthesis phase at both $128.7 \mathrm{nM}$ and $186.2 \mathrm{nM}$ concentrations, as well as an increase in G2/M and G0/G1 fragmented DNA. These data indicate that jararhagin is effective in reprogramming the cell cycle by inhibiting proliferation through synthesis inhibition.

The distinction between increased apoptosis or necrosis was assessed by the annexin V/PI labeling method by flow cytometry using the jararhagin treatment. Tanjoni (2005) demonstrated the induction of apoptosis with loss of cell adhesion, anoikis in jararhagin-treated endothelial cells, which indicates special attention to possible side effects on the vascular system [20].

There are several antiproliferative mechanisms, including apoptosis and senescence. Although senescence, similar to apoptosis, is also an extremely complex process, some forms of senescence are more precisely defined. Replicative senescence, which occurs in primary cells that are cultured in vitro, is dependent on telomere size and is usually reversed by telomerase expression. In recent years, however, it has become apparent that there is oncogene-induced senescence (OIS), which is activated by the activation of oncogenes such as Ras and Raf and requires p53, acting via CDK inhibitors, p21CIP, and p16INK. These two processes have irreversible arrest of growth and expression of beta-galactosidase in acid cell compartments in common, which allows the measurement of senescence. Senescent premalignant lesions were found in several normal tissues and are believed not to progress to malignancy due to senescence, indicating the importance of senescence as an antitumor process. This information, combined with the results obtained concerning p53 expression, demonstrates the participation of jararhagin concentrationdependent senescence and autophagy inducing mechanisms.

The ultrastructural microscopy images performed with the HEPA-1c1c7 tumor cells lineage obtained by confocal laser scanning fluorescence microscopy revealed at both concentrations a reduction in the number of morphologically viable cells, cell detachment, tumor parenchyma deconfiguration, cluster, and apoptotic body formation. While in the Control Group, an intense proliferation was observed with the formation of parenchymal structures. The expression of markers obtained in this lineage by confocal laser scanning fluorescence microscopy elucidated a significant dose-dependent increase in the expression of CD34 and CD44, glycoproteins involved in cell-cell interaction and function as an adhesion factor and cell migration, besides the considerable increase in activity of caspase-3, p53, and reduction in Cox-2 cyclooxygenase expression, actively involved in the process of tumorigenesis.

Scanning electron microscopy (SEM) showed hepatocellular carcinoma HEPA-1c1c7 cells cultured in monolayer and the extracellular matrix organization with many protrusions. Retractions and cell aggregates were observed at $128.7 \mathrm{nM}$ jararhagin concentrations. In cells treated with $186.2 \mathrm{nM}$, there was the detachment of the plaque surface and formation of smaller aggregates, apoptotic bodies, and necrotic debris.

The sum of IC50 values, morphological alterations, and cell viability together suggest the possible use of jararhagin as an antitumor agent capable of inducing cell death. However, their toxicity in normal cells should be evaluated and redirected to other studies, such as sitespecific nanocarriers to reduce the toxic effect in normal cells, as well as studies that elucidate the cellular signaling pathways involved in this process. After that it is possible to establish which are the inducers of necrotic and apoptotic death in normal cells, as they were done in the HEPA-1c1c7 hepatocarcinoma lineage, seeking to safely establish the use of this snake venom metalloproteinase in cancer therapy.

The HEPA-1c1c7 cells used in this study are murine hepatocarcinoma cells, and in jararhagin concentrations required for induction of IC $50 \%$ cytotoxicity were very close on a monometric scale, causing morphological and inductive alterations of similar human cells death. This may 
suppose an indication that murine cells, as well as human cells, are equivalently sensitive to the mechanism of action of jararhagin and can be used as efficiently as animal guinea pig models to prove the efficacy of this toxin in vivo.

\section{Conclusion}

Jararhagin-treated HEPA-1c1c7 murine hepatocellular carcinoma cells showed a reduction in the synthesis phase at both $128.7 \mathrm{nM}$ and $186.2 \mathrm{nM}$ concentrations, as well as an increase in fragmented DNA and arrestin G2/M. The expression of markers obtained in this lineage by confocal laser scanning fluorescence microscopy elucidated a significant dose-dependent increase in the expression of CD34 and CD44, glycoproteins involved in cell-cell interaction and that function as cell adhesion and migration factor, besides the considerable increase in activity of caspase-3, p53, and reduction of Cox-2 cyclooxygenase expression, actively involved in the process of tumorigenesis. Scanning electron microscopy (SEM) showed that HEPA1c1c7 hepatocellular carcinoma cells underwent significant morphological alterations such as cytoplasmic retraction and aggregate formation, also showing detachment of plaque surface and formation of apoptotic bodies and necrotic debris.

\section{References}

1. Institute National of Cancer - INCA. Liver cancer. 2018. Available in: <https://www.inca.gov.br/tipos-de-cancer/ cancer-de-figado $>$ Access on: 24 nov. 2019.

2. Lopes FLM, Coelho FF, Kruger JAP et al. Influence of Hepatocellular Carcinoma Etiology in the survival after resection. ABCD Arq Bras Cir Dig, 2016; 29 (2); 4:105108.

3. Balogh J, Victor D, Asham EH et al. Hepatocellular Carcinoma: A Review. Journal of hepatocellular carcinoma. 2016;3;13:41-53.

4. Caldwell S, Park SH. The epidemiology of hepatocellular cancer: from the perspectives of public health problem to tumor biology. J Gastroenterology. 2009; 44 (Suppl XIX);6:96-101.

5. Chedid MF. Hepatocellular carcinoma: diagnosis and operative management. ABCD Arq Bras Cir Dig. 2017; 30 (4); 7:272-278.
6. Institute Oncoguia. Liver Cancer survival rate. Available in:http://www.oncoguia.org.br/conteudo/taxa-de-sobrevidapara-cancer-de-figado/8295/208/> Access on: 21 nov 2019.

7. Ma R, Ravikiran M, Kwok H. Venon-based peptide therapy: insights into anti-cancer mechanism. 2017;8(59);23:100908-100930.

8. Ferreira BA, Deconte SR, Moura FBR et al. Inflammation, angiogenesis and fibrogenesis are differentially modulated by distinct domains of the snake venom metalloproteinase jararhagin. 2018;119;9:1179-1187.

9. Klein A, Capitanio JS, Maria DA, Ruiz IRG. Gene expression in SK-Mel-28 human melanoma cells treated with the snake venom jararhagin. 2011;57(1);8:1-8.

10. RodriguezMGP. Evaluation of antitumoreffects of ophidic metalloproteinase jararhagin in breast adenocarcinoma. 2012. 171 p. Masters thesis (Biothecnology) - Institute of Biomedical Sciences, USP. 2012;172:1-172.

11. Son DJ, Park MH, Chae SJ et al. Inhibitory effect of snake venom toxin from Vipera lebetina turanica on hormonerefractory human prostate cancer cell growth: induction of apoptosis through inactivation of nuclear factor $\mathrm{kB}$. Mol. Cancer Ther.2007;6;9:675-683.

12. Moura-da-Silva AM, Marcinkiewicz M, Niewiarowski S. Selective recognition of $\alpha 2 \beta 1$ integrin by jararhagin, a metalloproteinase desintegrin from Bothrops jararaca venom. Thrombosis Research. 2001;102;7:153-159.

13. Cimpeam AM, Ribatti D, Raica M. The chick embryo chorioallantoic membrane as a model to study tumor metastasis. Angiogenesis. 2008;11(4);9:311-319.

14. Correa MC, Maria DA, Moura-da-Silva AM, Pizzocaro KF, Ruiz IRG. Inhibition of melanoma cells tumorigenicity by the snake venom toxin jararhagin. Toxicon. 2002;40;10:739-748.

15. Simpson CD, Anyiwe K, Schimmer AD. Anoikis resistance and tumor metastasis. Cancer Lett. 2008;272(2);9:177-185.

16. Kenny PA, Lee GY, Myers CÁ et al. The morphologies of breast cancer cell lines in three-dimensional assays correlate with their profiles of gene expression. Mol. Oncol. 2007;1(1);13:84-96.

17. Do Amaral JB, Urabayashi MS, Machado-Santelli GM. Cell death and lumen formation in spheroids of MCF-7 cells. Cell Biol. Int.2010; 34 (3);8:267-274.

18. Li Q, Sato EF, Kira Y et al. A possible cooperation of SOD1 and cytochrome $\mathrm{c}$ in mitochondria-dependent apoptosis. Free Radic. Biol. Med. 2001;40(1);8:173-181.

19. Sodergren E. Lipid peroxidation in vivo. Evaluation and application of methods for measurement. Ph. D. thesis (Medical Science in Geriatrics) Comprehensive Summaries of Uppsala Dissertations from the Faculty of Medicine, Uppsala University, Scandinavia. 2000;78:1-78.

20. Tanjoni I, Weinlich R, Della-Casa MS et al. Jararhagin, a snake venom metalloproteinase, induces a specialized form of apoptosis (anoikis) selective to endothelial cell. Apoptosis. 2005;10;11:851-861. 